\title{
SERRATENE TRITERPENES FROM PINUS ARMANDII BARK
}

\author{
JIM-MIN FANG, WeI-YU TSAI and YU-ShIA ChenG* \\ Department of Chemistry, National Taiwan University Taipei, Taiwan 10764, Republic of China
}

(Received 19 July 1990)

Key Word Index Pinus armandit; Pinaceae; bark; triterpenes; serratene; nor-serratenone.

Abstract-Five known serratene triterpenes, a nor-serratenone and four new serratene derivatives have been isolated from the bark of Pinus armandii.

\section{INTRODUCTION}

Pinus armandii var. mastersiana, the armand pine, is an economically important conifer indigenous to the south west region of China [1]. We have recently reported flavonoids and stilbenes from the heartwood of this plant [2]. As a continuing study, we report here that serratenoid triterpenes are present in the bark of $P$. armandii. Serratenes have been mainly isolated from conifers (Pinaceae and Pinus species) and club mosses (Lycopodium species) $[3,4]$. They are a group of naturally occurring pentacyclic triterpenes biogenetically related to $\alpha$-onocerin [5]. The characteristic structural features of serratene triterpenes incorporate seven tertiary methyl groups (instead of eight methyls in common pentacyclic triterpenes), a central seven-membered $\mathrm{C}$ ring and a double bond between $\mathrm{C}-14$ and $\mathrm{C}-15$.

\section{RESLITS AND DISCUSSION}

The bark of $P$. armandii, collected in the Central Range of Taiwan, was exhaustively extracted with acetone. The concentrated extract was subjected to chromatography to give nine serratene triterpenes (1-9) in addition to a norserratenone (10). Compound $1, \mathrm{C}_{30} \mathrm{H}_{50} \mathrm{O}_{2}\left([\mathrm{M}]^{+} \mathrm{m} / z\right.$ 442.3793), is identified as serrat-14-en-3 $\beta, 21 \alpha$-diol [6]. The mass spectrum of 1 gave diagnostic intense fragments at $m / z 207(89 \%)$ and $220(78 \%)$ resulting from cleavage of the seven-membered $C$ ring, while fragments from retroDiels-Alder cleavage are minor [7]. On the basis of NMR evidence, both $3 \alpha-\mathrm{H}(\delta 3.17, d d, J=10,4 \mathrm{~Hz})$ and $21 \beta-\mathrm{H}$ $(\delta 3.22, d d, J=10,4 \mathrm{~Hz}$ ) are shown to be axial. Thus, rings $A$ and $E$ are considered to be in chair conformations with equatorially oriented $3 \beta$ and $12 \alpha$ hydroxy groups.

Compounds $2\left(\mathrm{mp} 305-308^{\circ}\right)$ and $3\left(296-298^{\circ}\right)$, both having $[\mathrm{M}]^{+}$at $m / z 442$, are recognized as isomers of 1 . The $\mathbf{H}-3$ in compound 2 appears to be axial (on the $x$-face) as inferred from its NMR resonance $(\delta 3.16, d d, J=11$, $5 \mathrm{~Hz}$ ), whereas the $\mathrm{H}-21$ is equatorial (on the $x$-face) occurring at $\delta 3.43$ as a broad singlet. On the other hand, both $3 \beta$ and $21 \alpha$ protons in 3 are equatorial as their NMR signals appear at $\delta 3.37$ and 3.43 as broad singlets. Thus, 2 and 3 are assigned as serrat-14-en-3 $\beta, 21 \beta$-diol and serrat14-en-3 $\alpha, 21 \beta$-diol, respectively [8].

Compound $4, \mathrm{C}_{31} \mathrm{H}_{52} \mathrm{O}_{2}$ (exact mass measurement, $\left[\mathrm{M}^{+}\right.$at $m / z 456.3988$ ) is a monomethoxy ether of 1 , which shows resonances at $\delta 3.33(s)$ and $2.60(d d, J=12$, $4 \mathrm{~Hz}$ ) attributable to the methoxy group and the axial geminal proton. As the ${ }^{13} \mathrm{C}$ signals for carbons in the $\mathrm{A}$ ring of 4 are substantially different from those of 1 , but carbons in the $\mathrm{E}$ ring remain unchanged (Table 1), the methoxy group is ascribed to the $3 \beta$ position. Thus, compound $4\left(\mathrm{mp} 318-319^{\circ}\right)$ is identified as $3 \beta$ methoxyserrat-14-en-21x-ol [8].

Compound $5,[\mathrm{M}]^{+}$at $m / z 498$, isolated for the first time from a natural source, shows IR absorption at $1720 \mathrm{~cm}^{-1}$ and ${ }^{1} \mathrm{H}$ NMR resonance at $\delta 2.02(3 \mathrm{H}, \mathrm{s})$ ascribed to an acetoxy group. This compound is $3 \beta$ methoxy-21 $\alpha$-acetoxyserrat-14-ene, an acetate of 4 , by chemical correlation.

Compound $6,[\mathrm{M}]^{+}$at $m / z 454$, contains a carbonyl group as indicated by the IR absorption at $1706 \mathrm{~cm}^{-1}$. The structure can be determined as $3 \beta$-methoxyserrat-14en-21-one [8] by analysis of its NMR spectra using the pulse techniques of DEPT, ${ }^{1} \mathrm{H}-{ }^{13} \mathrm{C}$ one-bond heteronuclear COSY and ${ }^{1} \mathrm{H}-{ }^{1} \mathrm{H}$ homonuclear COSY. According

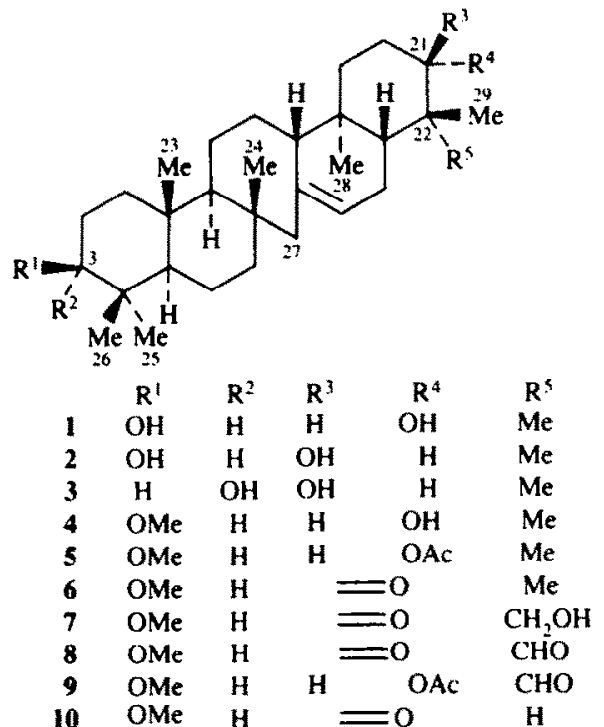


Table $1 .{ }^{13} \mathrm{C}$ NMR spectral data of serratenes $1-10\left(\mathrm{CDCl}_{3}, 75 \mathrm{MH} z\right.$, o)

\begin{tabular}{|c|c|c|c|c|c|c|c|c|c|c|}
\hline C & 1 & 2 & 3 & 4 & 5 & 6 & 7 & 8 & 9 & 10 \\
\hline 1 & 38.6 & 38.6 & 38.6 & 38.5 & 38.5 & 38.5 & 38.5 & 38.4 & 38.5 & 38.5 \\
\hline 2 & 25.2 & 25.2 & 25.2 & 22.4 & 22.3 & 22.3 & 22.4 & 223 & 22.4 & 22.3 \\
\hline 3 & 78.8 & 78.8 & 76.1 & 88.5 & 88.5 & 88.4 & 88.4 & 88.4 & 88.4 & 88.4 \\
\hline 4 & 38.2 & 38.2 & 37.7 & 38.2 & 38.2 & 38.2 & 38.2 & 38.2 & 38.2 & 38.2 \\
\hline 5 & 55.7 & 55.7 & 494 & 56.3 & 56.3 & 56.3 & 56.0 & 56.2 & 556 & 56.3 \\
\hline 6 & 18.9 & 18.9 & 18.8 & 18.8 & 18.8 & 18.7 & 18.7 & 18.7 & 18.6 & 18.7 \\
\hline 7 & 45.1 & 45.2 & 44.9 & 45.2 & 45.2 & 45.2 & 45.2 & 45.1 & 45.1 & 45.2 \\
\hline 8 & 39.0 & 39.0 & 38.2 & 38.9 & 38.9 & 38.9 & 38.9 & 38.9 & 38.9 & 38.9 \\
\hline 9 & 57.1 & 56.8 & 56.9 & 57.2 & 57.0 & 56.4 & 56.3 & 55.0 & 56.3 & 54.4 \\
\hline 10 & 36.1 & 36.0 & 35.9 & 36.1 & 36.0 & 36.1 & 36.1 & 36.6 & 36.1 & 36.1 \\
\hline 11 & 25.4 & 25.4 & 25.4 & 25.3 & 25.3 & 25.5 & 25.7 & 25.7 & 25.7 & 25.7 \\
\hline 12 & 27.6 & 27.2 & 28.4 & 27.7 & 27.2 & 27.2 & 27.3 & 272 & 27.2 & 27.3 \\
\hline 13 & 62.8 & 62.9 & 62.7 & 62.9 & 62.9 & 62.8 & 62.7 & 62.6 & 62.8 & 62.7 \\
\hline 14 & 138.2 & 138.5 & 138.6 & 138.3 & 138.3 & 138.3 & 138.5 & 138.6 & 138.1 & 138.6 \\
\hline 15 & 122.2 & 122.0 & 122.0 & 122.1 & 121.9 & 121.9 & 121.7 & 120.8 & 121.3 & 121.6 \\
\hline 16 & 24.0 & 24.0 & 24.0 & 24.1 & 24.1 & 24.4 & 24.6 & 24.6 & 24.4 & 29.5 \\
\hline 17 & 49.5 & 43.4 & 43.4 & 49.5 & 49.6 & 51.2 & 52.2 & 53.6 & 52.0 & 49.3 \\
\hline 18 & 38.9 & 37.1 & 37.3 & 38.9 & 37.1 & 37.1 & 37.1 & 37.1 & 37.1 & 37.1 \\
\hline 19 & 29.2 & 31.2 & 31.2 & 29.7 & 36.7 & 38.3 & 37.1 & 38.7 & 36.0 & 39.0 \\
\hline 20 & 27.2 & 27.5 & 27.1 & 27.2 & 23.8 & 34.7 & 35.4 & 37.1 & 24.2 & 38.0 \\
\hline 21 & 79.2 & 76.2 & 76.2 & 79.2 & 81.1 & 216.9 & 216.7 & 209.4 & 78.9 & 213.3 \\
\hline 22 & 37.1 & 37.4 & 37.4 & 37.1 & 37.7 & 47.6 & 52.8 & 63.2 & 52.5 & 45.6 \\
\hline 23 & 15.7 & 15.7 & 15.6 & 15.7 & 15.7 & 15.7 & 15.7 & 15.7 & 15.7 & 157 \\
\hline 24 & 19.8 & 19.8 & 19.8 & 19.8 & 19.8 & 19.8 & 19.8 & 19.8 & 19.8 & 198 \\
\hline 25 & 28.1 & 28.1 & 30.0 & 28.1 & 28.1 & 28.1 & 28.1 & 28.1 & 28.1 & 28.1 \\
\hline 26 & 15.4 & 15.4 & 13.5 & 16.2 & 16.2 & 16.2 & 16.2 & 16.2 & 16.2 & 16.2 \\
\hline 27 & 56.0 & 56.2 & 56.3 & 56.1 & 56.0 & 55.9 & 55.8 & 55.8 & $\$ 5.9$ & 56.0 \\
\hline 28 & 13.4 & 13.3 & 13.3 & 13.4 & 13.4 & 12.9 & 13.7 & 12.7 & 14.5 & 11.5 \\
\hline 29 & 275 & 27.7 & 21.8 & 27.5 & 27.5 & 24.5 & 20.7 & 16.3 & 21.1 & 11.0 \\
\hline 30 & 146 & 21.8 & 27.7 & 14.6 & 15.7 & 21.5 & 65.8 & 201.9 & 2046 & \\
\hline OMe &.- & & & 57.5 & 57.5 & 57.4 & 57.5 & 57.5 & 575 & $\$ 7.5$ \\
\hline \multirow[t]{2}{*}{$\mathrm{OAc}$} & & & - & & 21.3 & . & & & 20.2 & \\
\hline & & & & & 171.0 & & & & 170.5 & \\
\hline
\end{tabular}

to the octant rule [9], a negative Cotton effect in the CD spectrum of 6, $[\theta]_{286}-5000\left(\mathrm{MeOH} ; c^{7} 7.5 \times 10^{-3}\right)$, confirms the presence of a carbonyl group at $\mathrm{C}-21$.

Compounds $7\left(\mathrm{C}_{31} \mathrm{H}_{50} \mathrm{O}_{3}\right), 8\left(\mathrm{C}_{31} \mathrm{H}_{48} \mathrm{O}_{3}\right)$ and 9 $\left(\mathrm{C}_{33} \mathrm{H}_{52} \mathrm{O}_{4}\right)$ are novel serratene triterpenes. They show six tertiary methyl groups in their NMR spectra (Table 1). By meticulous analysis of the spectral data, compounds 7.9 are determined as $3 \beta$-methoxy-30-hydroxyserrat-14en-21-one, $3 \beta$-methoxy-21-oxoserrat-14-en-30-al and $3 \beta$ methoxy-21 $\alpha$-acetoxyserrat-14-en-30-al, respectively. To support the hydroxy group being on $\mathrm{C}-30\left(R^{5}\right)$ in 7 but not on $\mathrm{C}-29 .{ }^{1} \mathrm{H} \cdot{ }^{1} \mathrm{H}$ long range $\mathrm{COSY}$ reveals interaction between $\mathrm{H}-20 \alpha(\delta 2.66)$ and one of the $\mathrm{CH}_{2} \mathrm{OH}(\delta 3.57)$. A long range interaction of $\mathrm{H}-17$ and the aldehyde proton ( $\mathrm{CHO}$ ) in compound 8 was also observed. A negative Cotton effect in the $C D$ spectrum further supports 8 having the aldehyde group on the $x$-face. The stereochemical relationship in compound 9 is similarly elucidated. The acetoxy group is equatorial because its geminal proton (H-21) occurs at $\delta 4.72$ as a double of doublets ( $J$ $=11,5 \mathrm{~Hz}$ ). As compound 9 also shows a negative Cotton effect, its aldehyde group is also on the $x$-face according to the exciton coupling theory [10].

Compound 10, $\mathrm{C}_{30} \mathrm{H}_{48} \mathrm{O}_{2}\left([\mathrm{M}]^{+} m / z \text { 440.3629), [ } \alpha\right]_{\mathrm{D}}$ $-4.9^{\circ}\left(\mathrm{CHCl}_{3} ; c 1.4\right)$, is identified as an unusual norserra- tene, 30-nor-3 $\beta$-methoxyserrat-14-en-21-one, by comparison of its spectral data with those of an authentic sample [11]. The absence of the 30 -methyl group is consistent with a positive Cotton effect in the $\mathrm{CD}$ spectrum, $[\theta]_{282}$ $5400\left(\mathrm{MeOH} ; c 8.5 \times 10^{3}\right)$.

The occurrence of 30-norserratenone $10\left(R^{s}=H\right)$ is not yet fully understood. Finding the alcohol $7\left(R^{5}\right.$ $\left.=\mathrm{CH}_{2} \mathrm{OH}\right)$ and the aldehyde $8\left(\mathrm{R}^{5}=\mathrm{CHO}\right)$ in the same plant source suggests that 10 may be biogenetically derived from serratenone $6\left(R^{5}=M e\right)$ by a process of oxidative degradation via the intermediacy of 7 and 8 (Scheme 1).

\section{EXPERIMENTAI.}

Plant material. Bark of $P$. armundii Franchet var. mastersiana Hayata was collected on Tayulin mountain ( $2600 \mathrm{~m}$ altitude), Taichung, in August 1985. A voucher specimen, identified by Dr. Ta-Wei Hu, has been deposited in the Herbarium of the Taiwan Forestry Institute. The bark was air-dried $(760 \mathrm{~g})$ and extracted $\times 3$ with $\mathrm{Me}_{2} \mathrm{CO}$. The combined extracts $(37 \mathrm{~g}$ ) were subjected to $\mathrm{CC}$ on silica gel $(380 \mathrm{~g})$ by elution with the gradients of hexane and EtOAc. The components of each frs were combined for further purification by HPLC in either normal phase ( $\mu$-Porasil or LiChrosorb $\mathrm{Si} 60$ ) or reverse phase (L, $\mathrm{L}_{1}$ hrosorb RP-18) 


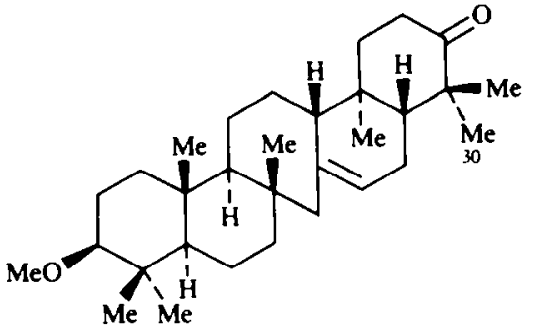

6<smiles>CCCC1(C)CCC(=O)[C@](C)(C=O)[C@H]1C(C)C</smiles>

8

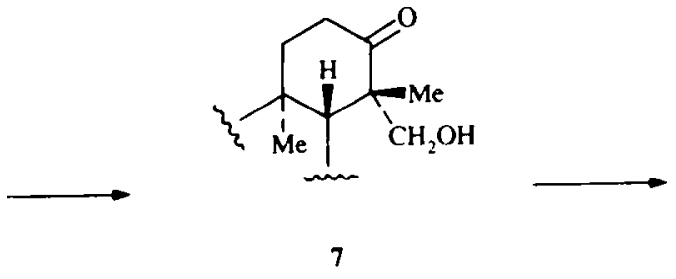

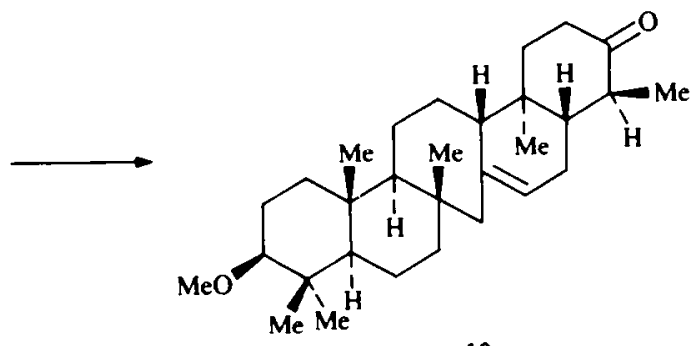

10

modes to give serratenes $5(13 \mathrm{mg}), 6(20 \mathrm{mg}), 10(28 \mathrm{mg}), 9$ (26 mg), 8 (29 mg), 4 (3.1 mg), 7(13.3 mg), $1(3 \mathrm{mg}), 2(800 \mathrm{mg})$ and $3(6.2 \mathrm{mg})$, according to the ascending order of polarity.

38-Methoxy-21x-acetoxyserrat-14-ene (5). $\mathrm{C}_{33} \mathrm{H}_{34} \mathrm{O}_{3}$, crys-

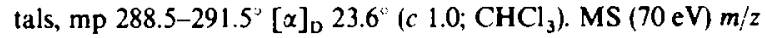
(rel. int.): $498[\mathrm{M}]^{+}(9), 483(4), 438\left[\mathrm{M}-\mathrm{HOAc}^{+}(3), 284(3)\right.$, 269 (10), 262 (28), 247 (5), 221 (83), 189 (68), 43 (100). HRMS: $[\mathrm{M}]^{+}$at $m / z$ 498.4044 (calcd 498.4072). IR $v_{\max }^{\mathrm{KBr}} \mathrm{cm}^{-1}: 1720,1250$, 1103, ' $\mathrm{H} \mathrm{NMR}\left(\mathrm{CDCl}_{3}, 300 \mathrm{MHz}\right): \delta 0.66$ (s, Mc-28), 0.72 (s, Me26), 0.77 ( $s, \mathrm{Me}-23), 0.78$ (2H, $m, \mathrm{H}-5, \mathrm{H}-13), 0.79(s, \mathrm{Me}-24), 0.82$ (s, Me-29), $0.84(1 \mathrm{H}, m, \mathrm{H}-1), 0.87$ (s, Me-30), $0.92(s, \mathrm{Me}-25), 1.10$ $(1 \mathrm{H}, m, \mathrm{H}-12), 1.14(2 \mathrm{H}, m, \mathrm{H}-11, \mathrm{H}-20), 1.18(1 \mathrm{H}, m, \mathrm{H}-7), 1.34$ ( $m$, $\mathrm{H}-17), 1.38(2 \mathrm{H}, m, \mathrm{H}-2, \mathrm{H}-7), 1.46(2 \mathrm{H}, m, \mathrm{H}-6), 1.50(2 \mathrm{H}, m, \mathrm{H}-$ 19), $1.60(1 \mathrm{H}, m, \mathrm{H}-20), 1.69(1 \mathrm{H}, m, \mathrm{H}-11), 1.73(1 \mathrm{H}, m, \mathrm{H}-27), 1.78$ (3H, $m, \mathrm{H}-1, \mathrm{H}-2, \mathrm{H}-9), 1.97(1 \mathrm{H}, m, \mathrm{H}-12), 2.02\left(\mathrm{~s}, \mathrm{MeCO}_{2}\right), 2.02$ $(2 \mathrm{H}, m, \mathrm{H}-16), 2.20(1 \mathrm{H}, b r d, J=15 \mathrm{~Hz}, \mathrm{H}-27), 2.60(d d, J=12$, $4 \mathrm{~Hz}, \mathrm{H}-3), 3.33$ (s, OMe), 4.48 ( $d d, J=11,5 \mathrm{~Hz}, \mathrm{H}-21$ ), 5.30 (br $s$, $\mathrm{H}-15$ ). Compound 5 was obtained as the exclusive product from acetylation $\left(\mathrm{Ac}_{2} \mathrm{O}\right.$, pyridine, $12 \mathrm{hr}$ at room temp.) of $3 \beta$ methoxyserrat-14-en-21 $\alpha$-ol (4).

3B-Methoxy-30-hydroxyserrat-14-en-21-one (7). $\mathrm{C}_{31} \mathrm{H}_{50} \mathrm{O}_{3}$, crystals, mp 226-228 $8^{\circ}$. $[\alpha]_{\mathrm{D}}-25.9^{\circ}\left(\mathrm{c} 0.89 ; \mathrm{CHCl}_{3}\right)$. MS (12 eV) $m / z$ (rel. int.): $470[\mathrm{M}]^{+}(10), 452\left[\mathrm{M}-\mathrm{H}_{2} \mathrm{O}\right]^{+}(5), 440$ (56), 408 (12), 323 (22), $234(13), 221$ (100), 219 (4), 204 (75), 189 (25). HRMS $[\mathrm{M}]^{+}$at $m / z 470.3769$ (calcd 470.3760). IR $v_{\max }^{\mathrm{KBr}} \mathrm{cm}^{-1}: 3441,1701$, 1102. ' ${ }^{1} \mathrm{H} \mathrm{NMR}\left(\mathrm{CDCl}_{3}\right): \delta 0.73(\mathrm{~s}, \mathrm{Me}-26), 0.76(\mathrm{~m}, \mathrm{H}-5), 0.78(\mathrm{~s}$, Me-23), 0.78 ( $m, \mathrm{H}-13), 0.81$ (s, Me-24), 0.85 (s, Me-28), 0.86 (1 H $m, \mathrm{H}-1), 0.93(\mathrm{~s}, \mathrm{Me}-25), 1.10(1 \mathrm{H}, m, \mathrm{H}-11), 1.14(1 \mathrm{H}, m, \mathrm{H}-12)$, 1.15 (s, Me-29), $1.18(1 \mathrm{H}, m . \mathrm{H}-7), 1.38(2 \mathrm{H}, \mathrm{m}, \mathrm{H}-2, \mathrm{H}-7), 1.44$ (m, $\mathrm{H}-6), 1.54(1 \mathrm{H}, m, \mathrm{H}-19), 1.72(1 \mathrm{H}, m, \mathrm{H}-11), 1.78(5 \mathrm{H}, m, \mathrm{H}-1, \mathrm{H}-$ 2 , H-9, H-17, H-27), 2.02 (2H, $m, \mathrm{H}-12, \mathrm{H}-16), 2.16$ (1H, m, H-19), $2.20(1 \mathrm{H}, b r d, J=15 \mathrm{~Hz}, \mathrm{H}-27), 2.38(1 \mathrm{H}, d d d, J=14,4,4 \mathrm{~Hz}, \mathrm{H}-$ $20 \beta), 2.60(d d, J=11,3 \mathrm{~Hz}, \mathrm{H}-3), 2.66(1 \mathrm{H}, d d d, J=14,14,6 \mathrm{~Hz}$, $\mathrm{H}-20 \alpha), 3.33(s, \mathrm{OMe}), 3.57\left(1 \mathrm{H}, d, J=7 \mathrm{~Hz}, \mathrm{CH}_{2} \mathrm{OH}\right), 4.00(1 \mathrm{H}, d$, $J=7 \mathrm{~Hz}, \mathrm{C}_{2} \mathrm{OH}$ ), 5.33 (brs, $\mathrm{H}-15$ ).
3ק-Methoxy-21-oxoserrat-14-en-30-al (8). $\mathrm{C}_{31} \mathrm{H}_{48} \mathrm{O}_{3}$, crys-

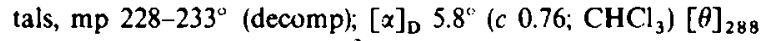
$-4800\left(\mathrm{MeOH} ; c 6.6 \times 10^{-3}\right)$. MS $(12 \mathrm{eV}) \mathrm{m} / 2$ (rel. int.): 468 $[\mathrm{M}]^{+}(7), 453(7), 438(8), 323(46), 284(3), 279(15), 232(4), 221$ (100), 217 (13), $189(40)$. HRMS: [M] ${ }^{+} 468.3621$ (calcd 468.3604). IR $v_{\max }^{\mathrm{KBr}} \mathrm{cm}^{-1}: 3427,1705,1102 .{ }^{1} \mathrm{H}$ NMR $\left(\mathrm{CDCl}_{3}\right): \delta 0.72(\mathrm{~s}, \mathrm{Me}-$ 26), 0.76 ( $m, \mathrm{H}-5), 0.77$ (s, Me-23), 0.80 (s, Me-24), 0.82 (m, H-13), 0.84 (s, Me-28), 0.84 (1 H, m, H-1), 0.93 (s, Mc-25), 1.08 (1 H, $m, \mathrm{H}-$ $11), 1.18(1 \mathrm{H}, m, \mathrm{H}-7), 1.22(1 \mathrm{H}, m, \mathrm{H}-12), 1.23(s, \mathrm{Me}-29), 1.38$ $(2 \mathrm{H}, m, \mathrm{H}-2, \mathrm{H}-7), 1.46(3 \mathrm{H}, m, \mathrm{H}-6, \mathrm{H}-19), 1.78(5 \mathrm{H}, m, \mathrm{H}-1, \mathrm{H}-2$, H-9, H-11, H-27), $1.90(3 \mathrm{H}, m, \mathrm{H}-12, \mathrm{H}-16, \mathrm{H}-17), 2.22(2 \mathrm{H}, m, \mathrm{H}-$ $19, \mathrm{H}-27), 2.32(1 \mathrm{H}, m, \mathrm{H}-16), 2.42(1 \mathrm{H}, m, \mathrm{H}-20 \beta), 2.60(d d, J=12$, $4 \mathrm{~Hz}, \mathrm{H}-3), 2.73$ (1H, ddd, $J=14,14,6 \mathrm{~Hz}, \mathrm{H}-20 x), 3.33$ (s, OMe), 5.34 (br s, H-15), 9.85 (s, C.HO)

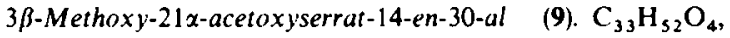
crystals, mp 230-232 (decomp.). $[\alpha]_{\mathrm{D}}-8.3^{\circ}$ (c $0.2 ; \mathrm{CHCl}_{3}$ ). $[\theta]_{286}-1600\left(\mathrm{MeOH} ; \mathrm{c} 3.9 \times 10^{-3}\right)$. MS (12 eV) $\mathrm{m} / z$ (rel. int.): $512[\mathrm{M}]^{+}(17), 452\left[\mathrm{M}-\mathrm{HOAC}^{+}{ }^{+}(50), 424(50), 323(25), 284(9)\right.$, 279 (25), 276 (6), 269 (6), 221 (100), 189 (42). HRMS [M] ${ }^{+}$at $m / z$ 512.3852 (calcd 512.3866). IR $v_{\max }^{\mathrm{KBr}} \mathrm{cm}^{-1}: 1724,1234,1102 \mathrm{~cm}^{-1}$. ${ }^{1} \mathrm{H}$ NMR $\left(\mathrm{CDCl}_{3}\right): \delta 0.61(\mathrm{~s}, \mathrm{Me}-28), 0.73(s, \mathrm{Me}-26), 0.78(\mathrm{~s}, \mathrm{Me}-$ 23), 0.75 (m, H-5), 0.78 ( $m$, H-13), $0.80(s, \mathrm{Me}-24), 0.89$ (1 H, $m, \mathrm{H}-$ 1), $0.93(s, \mathrm{Me}-25), 1.04(s, \mathrm{Me}-29), 1.08(m, \mathrm{H}-11), 1.13(1 \mathrm{H}, m, \mathrm{H}-$ 12), $1.18(1 \mathrm{H}, m, \mathrm{H}-7), 1.25(1 \mathrm{H}, m, \mathrm{H}-19), 1.32(1 \mathrm{H}, m, \mathrm{H}-19), 1.38$ (2H, m, H-2, H-7), $1.44(m, \mathrm{H}-6), 1.63(2 \mathrm{H}, m, \mathrm{H}-16, \mathrm{H}-17), 1.78$ $(4 \mathrm{H}, \mathrm{m}, \mathrm{H}-1, \mathrm{H}-9, \mathrm{H}-11, \mathrm{H}-27), 2.02\left(\mathrm{~s}, \mathrm{MeCO}_{2}\right), 2.02(2 \mathrm{H}, m, \mathrm{H}-$ $12, \mathrm{H}-20), 2.18(1 \mathrm{H}, m, \mathrm{H}-16), 2.20(1 \mathrm{H}, b r d, J=15 \mathrm{~Hz}, \mathrm{H}-27), 2.60$ $(d d, J=12,4 \mathrm{~Hz}, \mathrm{H}-3), 3.33(s, \mathrm{OMe}), 4.72(d d, J=11,5 \mathrm{~Hz} . \mathrm{H}-21)$, 5.25 (br s, H-15), 10.08 (s, CHO).

Acknowledgements - We are grateful to Dr Ta-Wei Hu (Department of Forestry, Chinese Culture University) for collection of plant material, to Dr Tian-Shung Wu (Providence College) for taking the $\mathrm{CD}$ measurements, to Dr Conner (Forest Products Laboratory, Wisconsin, U.S.A.) for the ${ }^{1}$ H NMR spectrum of 30 
nor-3 $\beta$-methoxyserrat-14-en-21-one and to the National Science Council (Republic of Chına) for financial support

\section{REFERENCES}

1. Li, H. L. (ed.) (1975) Flora of Taiwan Vol. 1, pp. 518-525. Epoch, Taiwan.

2. Fang, J. M., Su, W. C. and Cheng, Y. S. (1988) Phytochemistry 27, 1395.

3. Conner. A. H., Nagasampagi, B. A. and Rowe, J. W. (1984) Tetrahedron 40, 4217.

4. Cheng, Y. S., Chen, E. H. T. and Fang, J. M. (1975) J. Chin. Chem. Soc. (Taipei) 22, 341
5. Tsuda, Y.. Sano, T., Kawaguchi. K. and Inubushi, Y (1964) Tetrahedron Letters 1279

6. Inubush, Y., Sano, T. and Tsuda, Y. (1964) Tetrahedron Letters 1303.

7. Kutncy, J. P. and Eugendorf, G. (1969) Tetrahedron 25, 3753

8. Rowe, J. W. and Bower. C. L. (1965) Tetrahedron Letters 2745.

9. Moffitt. W., Woodward, R. B., Moscowitz. W. Klyne. W. and Djerassi. C. (1961) J. Am. Chem. Soc. 83, 4013.

10. Harada. N. and Nakanishi, K. (1983) Circular Dichroic Spectroscopy- Exciton Coupling in Organic Stereochemistry. Chap. 10 and 11. University Science Books, Mill Valley, CA.

11. Conner, A. H., Haromy, T. P. and Sundaralingam, M. (1981) J. Org. Chem. 46. 2987.

\title{
AGAVESIDE C, A STEROIDAL GLYCOSIDE FROM AGAVE CANTALA*
}

\author{
Girish C. Uniyal, Pawan K. Agrawal, † OM P. Satił and Raghunath S. Thakur
}

Central Institute of Medicinal \& Aromatic Plants, Lucknow 226016, India; ${ }_{+}$Department of Chemistry, University of Garhwal, Srinagar 246174 , India

(Receited 8 August 1990)

Key Word Index - Agate cantala: Agavaceae; fruits; steroidal glycoside; agavesıde C.

Abstract-A new steroidal glycoside, agaveside $C$, isolated from the fruits of Agave cantala was characterized as $3 \beta-\{\alpha-$

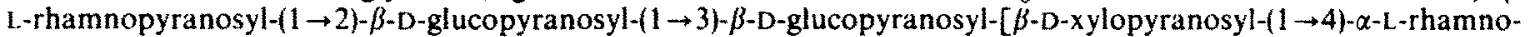
pyranosyl-(1 $(\rightarrow 2)]$ - $\beta$-D-glucopyranosyl $\}-2 \alpha$-hydroxy-25R-5 $\alpha$-spirostane on the basis of chemical degradation, ${ }^{13} \mathrm{CNMR}$ spectroscopy and fast atom bombardment mass spectrometry.

\section{INTRODLCTION}

In continuation of our chemical studies on Agave cantala [1]. we now report the isolation and structural elucidation of a steroidal glycoside, agaveside C (1).

\section{RESLLTS AND DISCUSSION}

The methanol extract of the fruits of A. cantala, on repeated chromatographic purification on silica gel columns afforded agaveside $\mathrm{C}(1)$. The glycosidic nature

*Part 34 in the series 'Studies on Indian Medicinal Plants'. For Part 33 see Jain. D. C., Agrawal, P. K. and Thakur, R. S. (1990) Planta Med. 56 (in press).

CIMAP Publication No. 936.

$\uparrow$ Author to whom correspondence should be addressed. of compound 1 was indicated by the broad absorption bands at 3397 and $1074 \mathrm{~cm}{ }^{1}$ in its IR spectrum and many resonances in the region $\delta 67-88$ in its ${ }^{13} \mathrm{C}$ NMR spectrum. Its spirostane skeleton was suggested by the occurrence of a resonance at $\delta 109.78$ in its ${ }^{13} \mathrm{CNMR}$ spectrum [2, 3] and supported by the IR absorption bands at 899 and $922 \mathrm{~cm}^{-1}$. The relative intensities of the two IR bands $\left(899 \mathrm{~cm}\right.$ ' $\left.>922 \mathrm{~cm}^{-1}\right)$ revealed $25 R$ stercochemistry which was consistent with the ${ }^{13} \mathrm{C}$ shielding data.

The $500 \mathrm{MHz}{ }^{1} \mathrm{H}$ NMR exhibited two singlets at $\delta 0.64$ and 0.76 and four doublets $(J=6.0 \mathrm{~Hz})$ at $\delta 0.75,1.03,1.06$ and 1.07 corresponding to two tertiary methyl groups at C-18 and C-19, and two secondary methyls at C.-21, C.-27 and $C-6$ of the rhamnopyranosyl units. The anomeric proton signals were observed at $04.78,4.80,5.05,5.08$, 5.17 and 5.44 and were correlated with ${ }^{13} \mathrm{C}$ resonances at $\delta 102.91,104.67,104.81,105.45,104.81$ and 104.97 respectively in a one bond $\mathrm{CH}$ correlation experiment, thus 